Horizons philosophiques

\title{
Détournement de capitaux et sublimation
}

Retour sur les lieux de la Critique de la raison cynique

\section{Jean-Pierre Couture}

Volume 17, numéro 2, printemps 2007

La pensée de Peter Sloterdijk

URI : https://id.erudit.org/iderudit/802634ar

DOI : https://doi.org/10.7202/802634ar

Aller au sommaire du numéro

Éditeur(s)

Collège Édouard-Montpetit

ISSN

1181-9227 (imprimé)

1920-2954 (numérique)

Découvrir la revue

Citer cet article

Couture, J.-P. (2007). Détournement de capitaux et sublimation : retour sur les lieux de la Critique de la raison cynique. Horizons philosophiques, 17(2), 1-20. https://doi.org/10.7202/802634ar d'utilisation que vous pouvez consulter en ligne.

https://apropos.erudit.org/fr/usagers/politique-dutilisation/ 


\section{Détournement de capitaux et sublimation \\ Retour sur les lieux de la Critique de la raison cynique}

Toute critique est un travail de pionnier dans le mal
du siècle ainsi qu'une partie de guérison exemplaire

L'étiquette de philosophe est chèrement conquise. Alors que l'on peut croire au caractère intrinsèquement philosophique d'un texte, une histoire sociale de la réception montre que l'étiquette "philosophie" obéit à des règles autant textuelles que sociales. Autrefois poète, prophète ou hérétique, le cas Nietzsche - devenu figure centrale de la philosophie professionnelle contemporaine - illustre de manière forte à quel point l'appellation contrôlée de la philosophie légitime a été un enjeu de haute lutte pendant trois générations ${ }^{1}$. La normalisation des lectures et des canons laisse toujours voir son jupon.

Nous célébrons cette année le vingtième anniversaire de la traduction française de la Critique de la raison cynique de Peter Sloterdijk. Un anniversaire partagé avec la traduction de la Théorie de l'agir communicationnel de Jürgen Habermas. Ces auteurs sont tous deux devenus les émules d'écoles et de sensibilités aujourd'hui bien distinctes. L'occasion est nommément rétrospective à l'égard de la genèse du phénomène Sloterdijk, car durant cette période il sera passé de simple "curiosité» au rang de "philosophe européen" récemment décoré du Prix Sigmund-Freud de l'Académie allemande. Faire retour ici sur les premières pages de l'œuvre inaugurale est d'un intérêt certain, car elles sont le théâtre d'un positionnement subtil dont la lumière rétrospective nous permet d'apprécier toute la mesure. De la parution de la Kritik ${ }^{2}$ en 1983 à l'affaire paneuropéenne entourant Règles pour le parc humain ${ }^{3}$ en 1999 , le combat symbolique visant à coller la bonne étiquette à l'auteur comme à l'œuvre constitue également l'occasion d'une enquête a posteriori sur les rouages de la réception. Tels sont les objets de ce texte.

À la sortie d'une crise qui le grandira et lui assurera définitivement un lectorat mondial, Sloterdijk proclame en 1999 - à la face du monde et de Habermas - la «mort de l'école de Francfort» et il 
s'assume dès lors comme renégat à l'égard de la "dictature sociale-libérale de la vertu4». Or, à l'égard de la présente occasion rétrospective, cette mort et cette rupture annoncées se donnaientelles à lire déjà en 1983? Ma réponse est sans équivoque : tout est déjà dit, mais discrètement sublimé, volatilisé ce qui a rendu méconnaissable, dans l'esprit du temps, la subversion latente de l'auteur. La critique de la critique qui s'y met en scène annonce, de plus, un détournement de capital culture ${ }^{5}$ pleinement consommé ultérieurement qui cherchera à retrouver les ressources d'une vraie critique par delà la théorie critique.

\section{Retour sur les lieux}

Peter Sloterdijk, né en 1947 à Karlsruhe en Allemagne, a étudié la philosophie, la germanistique et l'histoire à Munich et Hambourg avant de s'intéresser au structuralisme et à la pensée de Michel. Foucault. En 1976, il achève une recherche doctorale sur les écrits autobiographiques dans l'Allemagne de Weimar' et disparaît momentanément de la scène intellectuelle et définitivement du monde de la philosophie universitaire. Autour de 1980, il séjourne à Poona en Inde dans un ashram où s'est installée la secte de Bhagwan Shree Rajneesh qui y dirige un institut de recherche comparée sur les Religions ${ }^{7}$. Cette quête expérimentale auprès de ce "Wittgenstein de la religion" qui jouait à la religion pour la retourner contre elle-même lui fera dire que le maître de Poona demeure toujours, pour lui, l'une des plus grandes figures de pensée du $X X^{e}$ siècle ${ }^{8}$. Replongé dans la psychodynamique européenne dont les ressorts lui apparaissent avec une acuité nouvelle, "ça" aura commencé d'écrire la Critique de la raison cynique, confesse-t-il, au contact fortuit de l'insolence joviale et combien inspirante de Hannah Arendt?.

L'entrée en scène d'un nouveau venu est un moment qui révèle à la fois les contours de l'œuvre et la dynamique des positions parmi lesquelles elle tente de s'insérer. Ce sont des moments particuliers dans la vie d'un champ, lequel n'existe que par ses frontières qui policent la circulation des prétendants légitimes au jeu et qui en excluent les marginaux. Par la négative, on comprend qu'il y a un champ lorsque se manifeste cet enjeu du découpage et de l'exclusion. Plus généralement, il désigne le "système des relations de production, de circulation et de consommation des biens symboliques ${ }^{10}$ ", mais il agit non pas comme une externalité qui surdétermine ces derniers. Au contraire, le champ constitue une médiation qui donne à penser aux œuvres et qui les donne à lire. 
L'œuvre occupe en tout point cette position médiane qui fait d'elle tout à la fois un véhicule idéel et un reflet de l'état des lieux, car "(l'œuvre) possède la propriété de révéler, un peu à la façon d'un dispositif expérimental, les structures, les frontières et les enjeux du champ

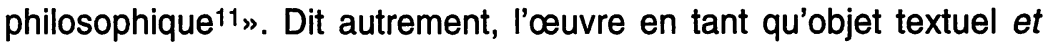
sociologique devient révélatrice de la structure de la société lectrice ${ }^{12}$ et même de l'état de censure de la société à qui elle se destine et à l'intérieur de laquelle elle peut faire sens (ou non) selon qu'elle reconduise le "monde réel» ou qu'elle soit, au contraire, tenue à l'écart comme «monde impossible».

Le philosophe, s'il est dépeint parfois comme un naïf qui tombe dans les puits à force de regarder au ciel, sait plus que quiconque ce que parler veut dire, c'est-à-dire qu'il sait exactement ce qu'il fait lorsqu'il utilise tel mot, lorsqu'il cite tel auteur. Alors qu'il choisit soigneusement ses habits de pensée, rien n'est laissé au hasard. Sur le point de faire son entrée costumée, les précautions de Sloterdijk à ces égards sont subtiles, car alors qu'il s'apprête à faire cette critique du cynisme contemporain, de qui reprendra-t-il la réplique et vers qui la retournera-t-il? Et de quelle critique s'agira-t-il?

\section{Savants patronages I}

Pour évoquer la thèse de cet opus éclectique de plus de 900 pages, dans sa version originale, rappelons que le diagnostic central de la Critique de la raison cynique établit que la modernité tardive a accouché du nec plus ultra du sujet moderne, le cynique, face auquel les ressorts habituels de la critique - démasquage, éducation, mobilisation - sont dépourvus. "(F)ausse conscience éclairée ${ }^{13}$ ", le cynisme brise les conditions candides du dialogue pacifiant de l'Aufklärung pour qui l'émancipation advient nécessairement par la mise à sac de l'ignorance. En tant que "cas limite de mélancolie, lequel parvient à contrôler ses symptômes dépressifs et à rester à peu près capable de travailler'14", le sujet politique de l'émancipation n'est plus intéressé de s'émanciper. Cette humeur diffuse, pierre d'achoppement d'une Aufklärung naïve et optimiste, sert de clé de voûte à l'homologie structurale que l'auteur propose avec l'Allemagne de Weimar comme idéal-type du cynisme catastrophile devenu aujourd'hui mentalité généralisée.

Ces diagnostics inauguraux de Sloterdijk sur le tournant cynique de la subjectivité moderne suggèrent que les ressorts de la critique doivent aujourd'hui renouer avec l'invective et la violence de la satire 
dont l'impulsion est aussi lointaine que la philosophie pantomimique de Diogène de Sinope, appelée kunisme. Face au mal du siècle, diagnostiqué comme le cynisme froid et routinisé de celui qui agit contre son intime conviction, la critique doit réincarner l'insolence chaude du démasquage du pouvoir qui la caractérisait également à l'aube de l'Aufklärung. C'est même là sa dernière chance. "Dans l'optique d'une critique de la raison cynique, l'Aufklärung peut renouveler sa chance et rester fidèle à son projet le plus intime : transformer l'être en être conscient ${ }^{15}$ ». Or, cette posture inaugurale d'une "rénovation" du projet des Lumières dans la Kritik n'est qu'une des tonalités de l'ambivalence de l'auteur envers l'Aufklärung. Mieux : il s'agit du masque d'Arlequin par lequel la voix de l'auteur exprime la subversion dans la continuité.

D'emblée, Sloterdijk se place effectivement sous le patronage de l'école de Francfort dans ce projet qu'il présente comme une actualisation du projet des Lumières. L'auteur fait sien le motif de la théorie critique chez T. W. Adorno - être concerné - à qui il reproche néanmoins "l'a priori de la douleur»16. Malgré son souci pour le monde, dit-il, la posture d'Adorno mène à un refus du monde. Le motif de la proximité s'éclipse à nouveau devant le pathos de la distance. C'est pourquoi - premier amendement - il faut retrouver avec Walter Benjamin, le marginal de l'école, l'esprit d'une critique comme immersion et proximité qui se fait voisine de son objet. "ll est impossible, dit-il, d'adopter un "point de vue" parce que les choses nous "sont tombées sur le dos"17". Conséquemment, la critique n'est pas «l'affaire d'une bonne distance, mais celle d'une bonne proximité".

Bien plus, ce retournement d'Adorno «sur ses pieds" concerne deuxième amendement - le fait qu'une critique authentique doive aller de la tête vers le corps ${ }^{18}$. La cérébralité émancipatrice doit se traduire en thérapie somatique. "Découvrir le corps vivant comme antenne du monde, c'est assurer son fondement réaliste à la connaissance philosophique du monde ${ }^{19}$ ». Dit autrement, ce sont les corps qui sont les sujets, les armes et le terrain de toute émancipation d'où la valeur de l'enseignement de la souveraineté joviale du kunisme pantomimique de Diogène, élevé au rang d'impulsion libératrice fondamentale chez Sloterdijk. Sur cette voie, le moment ardonien obligé - souffrir le mal du siècle - doit être dépassé dans l'état de félicité d'une science heureuse. C'est le mandat de la critique à laquelle souscrit Sloterdijk et à laquelle il demeurera fidèle jusqu'à aujourd'hui : "Toute critique est un travail de pionnier dans le mal 
du siècle ainsi qu'une partie de guérison exemplaire20». Un examen rapproché des prémisses de ces deux moments - diagnostic et thérapeutique - nous permet d'apprécier, plus avant, la sublimation de la mise à mort de Francfort - c'est-à-dire la manœuvre par laquelle cette idée "basse» et présentée sous un jour sublime - et le détournement de capital culturel qui investira la nouvelle critique.

\section{Critique de la critique. Sublimation et détournement I}

Rendre méconnaissable une pulsion inavouable, c'est euphémiser le discours par lequel une position nouvelle ou dominée souhaite déboulonner ou remplacer une position établie ou dominante. Sloterdijk amorce ainsi son parcours philosophique à titre de rénovateur de la théorie critique, symbole de la renaissance espérée du projet des Lumières. Fils de l'époque, il a eu à composer, comme tous ses contemporains, avec le rayonnement de la Dialectique de la raison d'Adorno et Horkheimer et de la théorie critique en général. Le thème de l'Aufklärung déviée de sa visée émancipatrice s'impose donc à tout prétendant au jeu. Mais alors qu'il revêt ce manteau conventionnel, Sloterdijk dresse un diagnostic dont la tautologie n'est qu'apparente, car la "mise en échec de l'Aufklärung par l'Aufklärung21" sape le lieu même de l'émancipation à laquelle la théorie de Francfort n'a pas renoncé, alors qu'elle ne comprend pas encore qu'elle perpétue insidieusement la maladie par la maladie.

D'abord, à suivre le diagnostic de l'auteur sur le patient, il n'y a plus de sujet politique de l'émancipation. Comme "sujet politique», le cynique sape les conditions du dialogue dont l'horizon moral prescrit une saine sortie de soi dans l'échange raisonné au terme duquel nos erreurs sont admises et notre adhésion à la raison, vivement consentie. Consciences durcies par une immunité réflexive qui "sait» déjà mais qui continue "quand même», les cyniques sont des "gens qui se rendent compte que le temps de la naïveté est révolu22». Émancipation? II n'y a personne au numéro que vous avez composé.

(Pis) encore, suivant la généalogie proposée par Sloterdijk, le cynique contemporain - terré dans cette forteresse inexpugnable est le sujet moderne réalisé des Lumières. Celles-ci se trouvent maintenant en face de leur créature inattendue qui a bien appris l'abc du réalisme, soit le devenir-forteresse cartésien fondé sur la peur de l'erreur, et qui affiche une compréhension toute militaire du devenirautonome kantien animé par la peur des liens et des appartenances qui «pensent à notre place». La naïveté de la croissance par le 
dialogue laisse place à des sujets autistes dont «les consciences sont plus dures que les faits ${ }^{23}$ ». En clair, pour le cynique au pouvoir, il vaut mieux détruire le monde plutôt que d'entrer en dialogue. «Hop là, c'est la vie!24», tel est le clairon de cette mobilisation tête baissée qui relie le cynisme au fascisme et qui autorise l'analogie entre le climat apocalyptique weimarien et le Zeitgeist de la guerre froide des années 1980 sur le point de muer en crise écologique contemporaine.

La critique de la raison cynique resterait un jeu de perles de verre universitaire, si elle n'examinait pas le rapport entre le problème de la survie et le danger du fascisme. En fait, la question de la "survie", de la conservation et de l'affirmation de soi, à laquelle tous les cynismes esquissent des réponses, cette question touche au problème central de la défense de l'existence et de la planification du futur dans les États nationaux modernes (...) où se rejoignent la peur de la désagrégation psychoculturelle, l'auto-affirmation régressive et la froideur rationnelle néo-positiviste, avec un courant vénérable de cynisme militaire (... 25 .

Au plan diagnostique, Sloterdijk invalide l'idée de l'Aufklärung qui guérit l'Aufklärung, du feu qui combat le feu, mais il souscrit encore à une contre-tradition de la raison censée redonner à la Raison l'impulsion qu'on ne lui connaît plus.

Sur ce plan, Francfort aurait encore tout à apprendre, car cette thérapeutique qui relève de la contre-tradition du "criticisme" est celle, thème abordé ci-haut, de l'émancipation des corps qui va de Diogène à Nietzsche jusqu'au féminisme du XXe siècle et celle de la satire capable de penser qui remonte aussi au philosophe du geste de Sinope et qui, un temps, a constitué le leitmotiv néo-kunique des marginaux du discours tels Marx, Nietzsche, Freud et aussi Heine dont Sloterdijk réclame le patronage en ces termes : «(II) a réussi le tour de force indépassé jusqu'à présent d'unir théorie et satire, connaissance et divertissement. Sur ses traces, nous allons essayer ici de réunifier les capacités de vérité de la littérature, de la satire et de l'art avec celles du "discours scientifique"26".

Dans l'optique d'un dépassement du cul-de-sac de Francfort, l'auteur ne tardera d'ailleurs pas en mettre en pratique ce dire vrai de la théâtralité des corps en faisant le récit de cette scène épique de la contestation étudiante de 1969 où l'émancipation tout intellectuelle de 
la théorie francfortoise - à l'exception des sympathies marquées de Marcuse - s'est vue sauvagement appropriée par la rue :

Peu de temps avant la mort d'Adorno, une scène eut lieu, dans une salle de cours de l'université de Francfort (...). Le philosophe était sur le point de commencer son cours lorsqu'un groupe de manifestants l'empêcha de monter sur l'estrade. Pareils incidents n'avaient rien d'extraordinaire en 1969. Dans ce cas précis, quelque chose nous oblige à y regarder de plus près. Parmi les perturbateurs, quelques étudiantes manifestèrent leur protestation en dénudant leurs seins devant le philosophe. D'un côté, la chair dénudée représentant la "critique» - de l'autre, l'homme amèrement déçu sans lequel presque aucun des assistants n'aurait appris ce qu'est la critique - le cynisme en action. Ce n'était pas la nudité de la violence qui rendait le philosophe muet, mais la violence de la nudité. (...). Là où des dissimulations sont constitutives d'une civilisation, là où la vie en société est soumise à la contrainte de mentir, apparaît dans l'énonciation réelle de la vérité un moment agressif, une dénudation mal à propos 27 .

Sloterdijk revient à cette scène plus tard, après avoir rebaptisé ce cynisme, kunisme, c'est-à-dire insolence plébéienne qui dans le dévoilement de la vérité n'a rien à perdre :

Chose ironique : c'est précisément Theodor W. Adorno, l'un des grands théoriciens de l'esthétique moderne, qui est devenu une victime de l'impulsion néo-kunique. (...) C'était, presque au sens antique, des corps dénudés kuniquement, des corps comme arguments, des corps comme armes. Indépendamment des motivations privées des manifestantes, la mise à nu de leurs seins comportait un axe de poussée antithéoriques. (...) Adorno était tombé d'une façon tragique et pourtant compréhensible dans la position du Socrate idéaliste et les jeunes femmes dans celle du récalcitrant Diogène. Des corps - espérons-le - intelligents se sont opposés avec obstination à la théorie la plus discernante ${ }^{18}$.

Que révèle ce récit anecdotique couplé au diagnostic général sur le cynisme des maîtres? Rien de moins qu'un dessein sublimé, celui de la mise au ban de l'école de Francfort au moyen d'un détourne- 
ment de capital culturel, c'est-à-dire par une manœuvre convaincue que la "vraie» critique vient d'ailleurs et qu'elle doit être subtilisée des mains d'une autre tradition. Sur ce plan, l'éthique francfortoise de l'émancipation par la mobilisation (se mobiliser contre l'injustice et l'ignorance) placée en face du cynique est une figure impuissante voire elle-même une figure de perpétuation du cynisme : non seulement n'y-a-t-il plus personne à mobiliser (mauvais numéro), mais le principe de la mobilisation elle-même participe de la logique de l'hégémonie que l'on souhaite combattre. "La prétendue politisation part d'une militarisation plus intensive et d'une mobilisation $s$ tratégique des consciences, et cela pas seulement en surface. Elle pénètre profondément jusque dans l'attitude corporelle et les formes de la perception 29 ». La théorie critique n'est pas critique de la mobilisation politique.

Par delà le récit de cet épisode où Sloterdijk formule une invective feutrée envers ses maîtres, on apprend aussi que la véritable critique vient d'ailleurs, que la guérison doit emprunter d'autres chemins, que le capital culturel et les ressources intellectuelles de la pensée critique actualisée se trouvent par delà la théorie critique dont "(l)'élément masochiste a surpassé l'élément créateur ${ }^{30}$ ". Si Sloterdijk tient en haute révérence les figures de Nietzsche et Heine dans les premières pages de la Kritik, en raison de leur impulsion néo-kunique, c'est parce qu'ils sont - d'une part, thème mineur - les propagateurs d'une littérature capable de vérité qui va au-delà du compromis de «l'essai». Cette littérature "centauresque»31 qui se présente sur scène dans le double habit de l'art et de la pensée, Sloterdijk en fera plus tard le thème-clé de la compréhension de l'œuvre de Nietzsche lui-même ${ }^{32}$ pour ne pas dire de la sienne propre. Or, ces savants patronages révèlent - d'autre part, thème majeur - que l'esprit de "rénovation" de la théorie de Francfort et de la gauche est beaucoup plus radical qu'une simple permissivité dans la forme expressive du discours, il s'agit plutôt, thème inavoué, de détourner la source de la critique ou plutôt de la restituer dans sa "véritable tradition" contre le monopole de Francfort. Le maître discret sort de sa tanière; on ne pourra réfléchir à neuf sur le monde sans compter sur Heidegger :

Sans le savoir et, pour une bonne part, même sans vouloir le savoir (en Allemagne, qui plus est, avec une résolution furieuse de ne pas l'admettre), la nouvelle gauche est une gauche existentialiste, une gauche néo-kunique, je risque l'expression : une gauche heideggerienne ${ }^{33}$. 
Ce qui ne se donne pas à lire ici, alors que Sloterdijk soulève discrètement son masque à sa sortie de scène, c'est la formule programmatique de cette "hypothèse" qui deviendra en fait le lieu de parole et de pensée, en propre, pour le nouveau philosophe. Une sorte de prolégomènes à toute politique future qui en l'état de sa formulation initiale ne fait que questionner "un tabou presque

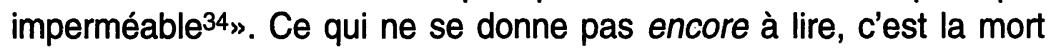
de Francfort.

\section{Savants patronages II}

Que l'on me permette de déroger, un temps, au commentaire de la Kritik pour démontrer que ce visage programmatique discret ne prendra pas de temps à s'affirmer dans l'œuvre. Avec La mobilisation infinie ${ }^{35}$, on comprend beaucoup mieux ce que Sloterdijk semait à titre exploratoire et timide au détour de ce commentaire sur Heidegger. Cette deuxième Kritik, celle de la "cinétique politique», a dans sa mire la modernité et la théorie critique elle-même, car elles sont toutes deux incapables de réfléchir à l'essentiel des problèmes de notre temps. "Ce qui avait l'air d'être un départ contrôlé vers.la liberté s'avère être une glissade vers une hétéromobilité catastrophique et incontrôlable ${ }^{36}$ ". À la recherche d'un réel principe critique qui jugerait du "dehors" le continuum de la crise moderne, Sloterdijk ne souhaite ni applaudir ni désespérer devant l'époque :

Une critique de ce type brise l'échouement cynico-mélancolique au monde qui se présente aujourd'hui partout comme consentement postmoderne. Mais elle évite aussi la contemplation masochiste de la totalité qui conduit au marginalisme métaphysique. Ni partisane du refus de s'engager ni consentante, la critique alternative a pour objet de promouvoir une théorie critique de l'être-au-monde ${ }^{37}$.

L'amalgame n'aura pas échappé au fin lecteur. Sloterdijk plaide, en 1989, pour un dialogue entre théorie critique et phénoménologie heideggerienne. En revisitant Francfort via Fribourg, Sloterdijk lance fatalement que la dernière est le vrai siège d'une critique de la mobilisation moderne. Un éclair déchire le ciel à Karlsruhe : «il n'y a plus en cette matière de théorie critique de Francfort, mais bien une de Fribourg 38 ". Critiquer la fausse "conscience éclairée mobilisée»39, c'est identifier la mobilisation contemporaine à un nihilisme de la volatilisation du monde qui est le versant immanentiste de l'ancienne haine métaphysique du monde. Si Heidegger conduit sur un chemin 
qui serait le point d'Archimède de la critique, c'est qu'il nous mène sur le chemin d'une "phénoménologie de la venue-au-monde» 40 , sur le chemin d'une anthropologie du "lieu» humain subsumé par la métaphysique de l'au-delà et liquéfié par le processus de la mobilisation, thématiques dont les échos résonneront d'ailleurs jusque dans le projet des Sphères ${ }^{41}$ chez Sloterdijk.

La rupture annoncée est consommée. Lier Heidegger à la théorie critique constitue un outrage qui néglige sciemment le fait que Francfort s'est constituée contre Fribourg : en 1931, dans sa leçon professorale inaugurale ${ }^{42}$, Adorno, symbole d'un renouveau du marxisme aux côtés de Horkheimer, amorce sa carrière philosophique par une attaque de Heidegger qu'il rend responsable du déclin de la philosophie, une critique qu'il poursuivra pendant plus de trente ans sans jamais d'ailleurs jouir d'une réplique de la part du destinataire. Pour les émules de l'école, il s'agira d'un rite obligé. Habermas se commet publiquement contre Heidegger, en 1953, dans un texte qui incarne son tout premier positionnement philosophique public ${ }^{43}$, un art qu'il maîtrisera assurément dans les décennies à venir et qui fera montre de la permanence de la critique des courants issus du continuum Nietzsche-Heidegger auquel il associe, lui, le «postmodernisme». La tentative de faire dériver la critique authentique à partir de ces auteurs maudits - latente en 1983, définitive à partir de 1986 (Nietzsche) et 1989 (Heidegger) - expose Sloterdijk aux tirs nourris des gardiens de la pensée officielle qui s'assure de maintenir, dans l'état du champ, l'impossibilité de militer pour un tel dialogue entre Adorno et Heidegger, même si Karlsruhe ${ }^{44}$ se situe à l'exact milieu géographique entre Fribourg et Francfort...

À l'égard de mon propos, les positionnements inauguraux de Sloterdijk nous renseignent sur la subtilité requise dans la mise en scène et l'expression de pulsions subversives à l'égard des discours établis. Se faire passer, par la main gauche, pour un héritier fidèle d'une tradition dont on subvertit, par la main droite, l'essence et le fondement, voilà une manœuvre qui permet d'être lu et reçu, mais aussi de désobéir à la sublime récapitulation de la tradition. Pour avoir voulu replacer la critique dans sa tradition dite originelle, Sloterdijk s'est prêté au jeu de ces transfuges «qui investissent un grand capital spécifique dans la subversion de l'ordre sacerdotal et qui puisent dans une lecture rénovée des sources les plus consacrées les armes d'une révolution destinée à restaurer la tradition dans son authenticité originaire ${ }^{45}$ ». Mais, puisqu'il faut y revenir, qu'y a-t-on lu en 1983, 
alors que cette pensée en action ne se laissait pas deviner par la lumière rétrospective?

\section{Réceptions et polémiques ou «qu'est-ce que de la philosophie?"}

Critique de la raison cynique en tant que pièce inaugurale de l'édifice sloterdijkien, n'est pas seulement porteuse des grandes intuitions de l'œuvre, mais aussi des ingrédients de toute la réception ultérieure. Alors qu'à l'été 1983, en France, on s'interroge sur le "silence des intellectuels", la presse culturelle allemande se déchire, elle, sur l'étiquette que devra porter ce nouveau venu des livres à-laSuhrkamp sur l'esprit du temps.

Dans son étude fort instructive, Otto Kallscheuer fait remarquer que deux mois après sa parution, en mars, la Kritik exige de tous les intervenants de la presse culturelle qu'ils se situent par rapport à l'événement qui, faut-il le rappeler, a fait écouler 50000 exemplaires dans les mois suivants sa parution, 70000 vers avril 1984 et 120000 au moment de sa traduction française en 1987 (du jamais vu dans le monde l'édition pour un titre «philosophique»). Évidemment, le succès commercial ne fait pas d'un livre une œuvre importante, laquelle doit réussir à réorganiser les positions dans le champ, c'est-à-dire se hisser parmi les quelques discours qui forment et épuisent la doxa de l'époque. Or, le "succès commercial» et l' «œurre importante" ne sont pas non plus des antipodes, car le rôle de la critique littéraire et de ses points de vue concurrents (véritable "querelle des recensions") a en retour "des "effets externes" positifs sur le marché économique du livre 46 ", c'est dire que le moment de l'étiquetage est décisif pour relier l'œuvre à son statut, pour l'endiguer dans le circuit de circulation conforme à son rang.

À l'été 1983, la réception de la Critique de la raison cynique est une manifestation exemplaire de ce travail d'étiquetage et des dissensions qu'il soulève. L'œuvre a effectivement généré un combat symbolique portant sur les frontières de la "légitimité" à être ou ne pas être "philosophiquement recevable" parce qu'elle se situait - ô malheur - à la frontière du succès commercial et de l'académie, du langage commun et de la théorie. Pour le comprendre de manière idéale-typique, Kallscheuer rappelle que la "pertinence culturelle" d'une œuvre, sa respectabilité, ne peut pas s'imposer seulement par la sanction du «marché», car elle doit avant tout être reconnue par l'«académie» (instance de consécration scientifique) ou 
par le "milieu» (instance de consécration des initiés). Pour le nouveau venu ou l'outsider, la carrière escomptée se dessinera entre la pertinence ou l'impertinence culturelle, entre le seul marché du livre ou la reconnaissance par les pairs, catégories qui tendent à s'exclure mutuellement au sens où le succès dans l'une des structures annule souvent le succès possible ou escompté dans l'autre.

Dans le jeu de cette mécanique, Sloterdijk a porté tous les chapeaux. Il a été à la fois sacré auteur d'un chef-d'œuvre philosophique et d'un livre sans arguments, d'un brûlot sur la pensée 68 et d'une lecture de plage. Le litige entre les recenseurs atteignit même ce point où ils ne se sont pas privés d'utiliser les ressorts de l'insulte pour conquérir le monopole du jugement culturel légitime 47. L'hybridité du texte - satire et science, prose et argumentation, images et textes - est indéniablement le thème fort de cette querelle : on n'arrive pas à s'entendre sur le statut de ce texte newlook. Cela n'a pas échappé d'ailleurs à Jürgen Habermas qui se garde d'entrer dans le cercle de l'injure et qui y va de son propre diagnostic nuancé sur le premier livre de Sloterdijk qui d'emblée, selon lui, "mérite sa notoriété rapidement acquise 48 ". La spécificité de la Kritik tient au fait qu'elle se situe entre la philosophie et l'essai, une catégorie culturelle intermédiaire "plutôt commune en France" qui révèle ici «un flot de pensées très colorées avec une touche de sérieux germanique lorsqu'il touche à la substance ${ }^{49}$ ». Malgré ses réserves à l'endroit d'un kunisme qui lui apparaît être un cul-de-sac de la critique qui est à la remorque de son opposant, Habermas précise que «Monsieur Sloterdijk n'est pas un néoconservateur» même s'il le situe non sans lucidité entre Heine et Heidegger.

Non sans lucidité, peut-être, mais nous sommes loin des inimitiés de 1999 lors desquelles le même Habermas a conduit, dans l'ombre, une vendetta contre l'auteur "fascistoïde" de Règles pour le parc humain. Non, dans cette recension le dessein subversif du nouveau venu passe inaperçu alors que son but est précisément de miner le statut de celui qui le lit ici avec empathie. Sloterdijk est des "nôtres", mais la suite allait décrire un autre scénario...

Dès lors qu'il aura parlé de "sélection" dans sa réflexion sur l'anthropotechnique (l'homme comme "éleveur» de l'homme qui prend actuellement le visage inquiétant d'une intervention "anonyme» sur le génome humain), Sloterdijk provoquera une énorme tempête polémique à l'allemande, avec ses ramifications françaises, initiée par 
une partie de la presse voulant conjurer le spectre d'une banalisation de l'eugénisme ${ }^{50}$. L'architectonique de cette polémique - dans laquelle Habermas refuse d'entrer en dialogue avec Sloterdijk ${ }^{51}$ n'est que l'aboutissement éclatant d'un conflit latent entre position établie et position dominée dans le champ et elle fait montre de cet enjeu par lequel on refuse ou confère au penseur le statut de philosophe et donc d'interlocuteur valable. Pour sûr, dans ce moment-pivot où il déclare la mort de la théorie critique, Sloterdijk a contribué encore davantage à ébruiter l'existence de son œuvre à l'étranger, surtout en France, où l'étiquette de philosophe respectable lui est, depuis, naturellement conférée. Au terme de l'«affaire»52, force est d'admettre que Sloterdijk a su se hisser parmi les positions incontournables dans le paysage philosophique européen. Dans le marché français, la diffusion massive de la conférence Règles pour le parc humain via les Éditions Mille et une nuits, la réédition subséquente de la Critique de la raison cynique et du Penseur sur scène en 2000 et la parution de nouvelles traductions jusque-là laissées en plan en sont les traces.

L'exemple initial de la réception de la Critique de la raison cynique montre que "la légitimité culturelle n'est pas une catégorie fixe et irrévocable une fois pour toutes, mais bien plutôt un lieu de combat 53 ". Dans le cas de Sloterdijk, ce combat sera permanent, rappelle Tuinen, car «à partir de ce moment et jusqu'à aujourd'hui, une sorte de controverse permanente est mise en branle

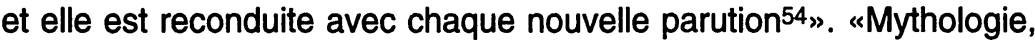
nicht Philosophie". Mais pourquoi? Si Sloterdijk mène un combat en propre, c'est celui en faveur du rôle de l'intellectuel public comme animateur de la démocratie et comme pont entre langage commun et philosophie. La hardiesse de cette entreprise à l'égard de la très sérieuse philosophie de chaire devient médiatique : Sloterdijk a investi le médium télévisuel où il co-anime mensuellement Das philosophische Quartett, un débat intellectuel sur une question d'actualité. Clownerie pour certains, action conséquente de celui qui n'a pas qu'une théorie critique des médias, pour les autres, autant d'épithètes qui sont une variation sur le thème de l'établissement des frontières de la production légitime voire du comportement légitime, car à la parution de chaque opus de l'édifice sloterdijkien et à chaque scandale qui le suit, on se demandera, mais qu'est-ce que de la philosophie? 


\section{Exit. Gauche terrestre ou gauche gazeuse? Sublimation et détournement II}

Tout ce qui est solide se volatilise

Marx

Fort d'une consécration de mieux en mieux assurée, Sloterdijk, philosophe européen, poursuit toujours sur sa lancée inaugurale et centauresque en alliant dans un même projet de pensée, l'art et la philosophie. Cet impératif esthétique qui relie la forme et le fond, le contenant et le contenu lui a conféré les honneurs de l'Académie allemande en 2005, moment où l'auteur affirme, plus avant, sa stratégie esthétique.

Ce qui s'esquisse, selon moi, dans la remise du Prix SigmundFreud pour la prose scientifique est un geste culturel et politique significatif. Elle soutient implicitement, si je comprends bien, l'idée que la littérarité est constitutive également de l'articulation des savoirs contemporains (...). Cela signifie que la question de la forme langagière d'un savoir ne peut jamais demeurer complètement en dehors du savoir lui-même. Ce qui prête chaque fois à controverse est le degré jusqu'où l'on pourra pousser l'individuation rhétorique dans le discours théorique - je me permets ici, à titre de témoin et d'objet de telles controverses, de signifier que je n'ai jamais douté de l'appartenance de mes travaux au monde de la connaissance et de la science même s'ils sont apparentés à l'occasion à une forme littéraire ou mieux extradiscursive 55 .

De 1983 à nos jours, Sloterdijk est certainement le créateur d'une œuvre qui a pour dessein de ne pas laisser l'éros philosophique disparaître au profit unique de la sobriété d'une scientificité morne. Cette position ne s'en prend ni aux producteurs de savoir ni à la science, car la facture boulimique de l'œuvre se nourrit de l'apport des découvertes scientifiques (y compris biologiques) auxquelles Sloterdijk redonne une assise phénoménologique ou narrative ${ }^{56}$. Sa militance a trait plutôt à la raréfaction du rôle public de la philosophie qui doit retrouver sa voix propre parmi les vues sur le monde.

Or, au sortir de scène, il me faut préciser que cette apparente humilité ne doit pas occulter le fait que la démarche de Sloterdijk a beaucoup plus à voir avec une «mégalopathie» revendiquée 57 , être le "patient des grandes questions», soit une méthodologie qui prescrit 
qu'il nous faut traiter du Grand, dit-il, puisque nos problèmes sont énormes.

Sur cette voie, la Grande exagération - le projet des Sphères est nommément un projet de réécriture de l'histoire, ici morphologique, de l'humanité - serait la marque d'une philosophie conforme à l'air du temps 58 . Le monstrueux de l'époque se fait monstrueux littéraire, son style revêt la forme de l'essai et recourt à l'hyperbole, son objet est l'excès et l'abondance comme donne fondamentale de la condition occidentale contemporaine. Quitte à confronter une gauche qui ne puisse imaginer une politique sans politisation de la misère, la volatilisation du monde par le capitalisme avancé, tel qu'anticipée par Marx, rappelle Sloterdijk, dissout le matériel dans l'air. La misère globale, sublimée par le confort local.

À la toute fin du Palais de cristal - une phénoménologie contemporaine de l'archipel capitaliste clos sur lui-même - Sloterdijk passe en revue les éléments d'une irréductible appartenance humaine au lieu et au temps. II soutient qu'une gauche terrestre, à la différence de la gauche céleste de l'universalisme abstrait, doit se détourner de ses anciennes idoles et se réintéresser aux

\section{(...) caractéristiques inhérentes à l'infrastructure du devenir dans des sphères humaines réelles. Elles comptent au nombre des caractéristiques l'être-là fini, concret, immergé et capable de transmettre. Pour faire encore une fois appel à la façon de parler de l'ontologie : l'être-étendu sur son propre lieu est la bonne habitude de l'être ${ }^{59}$.}

L'esthétique mégalopathique et le point de vue du narrateur-DieuSloterdijk sont ainsi détournés de leur cours pour s'intéresser, à beaucoup plus petite échelle, au lien et au lieu qui tissent et engendrent le fait humain. Le grand discours narratif, en guise de remarque critique, malgré ses grandiloquences initiales qui pouvaient laisser croire à l'édification d'une Grande Politique, se volatilise devant l'irréductible couveuse humaine, point aveugle de toute politique des grandeurs, élevée ici au rang d'intermédialité fondamentale. Pour Henk Oosterling, ce nécessaire downsizing ramène l'énormité à sa juste proportion :

L'hyperpolitique mégalopsychique devient un inter-esse micropolitique. En ramenant et en miniaturisant cette mégalopsyché à ses proportions «médiologiques" [ce qui nous 
relie], la stratégie politico-esthétique de Sloterdijk se comprend mieux à titre de micropolitique de l'espace public, c'est-à-dire l'art comme espace public 60 .

Façon de rappeler utilement que la "critique» et la "politique" chez Sloterdijk sont sublimées, volatilisées dans une philosophie de l'espace commun. Ainsi, l'auteur confirme-t-il moins son attrait pour une Grande Politique que pour la retraite épicurienne esthétisée, la gauche gazeuse assumée.

L'attitude de Sloterdijk envers la politique est donc fort ambivalente, et ce, même s'il traite de catégories politiques dans la plupart de ses œuvres. En fait, l'étiquette de "penseur politique" est déclinée en propre par l'auteur, car il y associe d'emblée une connotation schmittienne qui exige du politique penseur qu'il se désigne un "ennemi" lequel exige "chaque matin un briefing de guerre, chaque matin des observations sur les opérations ennemies 61 ». II ajoute : «Je ne conçois pas la politique comme la conduite de la guerre par les moyens de la paix. En ce sens, je ne suis pas un penseur politique».

\author{
Jean-Pierre Couture * \\ Doctorant en science politique, UQAM
}


1. Louis Pinto. Les neveux de Zarathoustra, Paris, Seuil, 1995.

2. Peter Sloterdijk. Kritik der zynischen Vernunft, Frankfurt am Main, Suhrkamp, 1983.

3. Peter Sloterdijk. Règles pour le parc humain, trad. Olivier Mannoni, Paris, Mille et une nuits, 1999

4. Peter Sloterdijk. "Die Kritische Theorie ist tot», Die Zeit, 9.9.99. Ma traduction.

5. Dans ce texte, la notion de "capital culturel», dans une acception bourdieusienne, désigne les éléments d'une ressource symbolique, soit les canons littéraires ou philosophiques et les prestiges - titres, diplômes, positions - qui leur sont conférés ou associés et dont on se dispute le contrôle, la possession ou l'incarnation légitime. En tant que capital symbolique, assujetti à la reconnaissance sociale, il est l'objet d'une lutte qui structure la joute intellectuelle et artistique. "Du fait que le capital symbolique n'est pas autre chose que le capital économique ou culturel lorsqu'il est connu et reconnu, lorsqu'il est connu selon les catégories de perception qu'il impose, les rapports de force symbolique tendent à reproduire et à renforcer les rapports de force qui constituent la structure de l'espace social» (Pierre Bourdieu, Choses dites, Minuit, 1987, p. 160).

6. Peter Sloterdijk. Literatur und Organisation von Lebenserfahrung, München, Hanser, 1978.

7. Né Rajneesh Chandra Mohanin en 1931, "Baghwan» (le « divin ") a enseigné la philosophie et la psychologie à l'Université de Jabalpur. Son ashram de Poona a attiré de 1974 à 1981 des dizaines de milliers d'Occidentaux. Des suites de son déménagement en Oregon, Baghwan devenu Osho a montré son visage sombre et est devenu le gourou d'une secte oppressive à laquelle John Updike a consacré le roman intitulé "S". Après ses démêlés avec le FBI, le gourou retourna à Poona où il mourut en 1990 (Holger Frhr. von Dobeneck. Das Sloterdijk Alphabet, Würzburg, Königshausen \& Neumann, 2002, p. 24-26). Sloterdijk s'est confessé à au moins deux reprises (Essai d'intoxication volontaire, trad. Olivier Mannoni, Paris, Calmann-Levy, 2000; Ni le soleil ni la mort, trad. Olivier Mannoni, Paris, Pauvert, 2003) sur cet épidode extatique non sans nostalgie contenue pour ces communes brûlantes, ces couveuses et psychoréacteurs qui défient l'atomisation marchande. Refroidi, il admet que l'esprit de la secte encourt toujours le risque d'un délire paranoïaque ou gouroucratique qui n'a pas épargné, non plus, Jacques Lacan.

8. Sjoerd van Tuinen. Peter Sloterdijk. Ein Profil, Paderborn, Wilhelm Fink Verlag, 2006, p. 17.

9. Peter Sloterdijk. Critique de la raison cynique, trad. Hans Hildenbrand, Paris, Bourgois, 1987, p. 309.

10. Pierre Bourdieu. "personne", «légalement» et «impudence», là où, dans l'édition, se trouve, respectivement, "pensée», «également» et cimprudence" "personne», «légalement» et «impudence», là où, dans l'édition, se trouve, respectivement, "pensée», "également» et «imprudence» "Le marché des biens symboliques», L'année sociologique 22, 1971, p. 49.

11. Louis Pinto. Les neveux de Zarathoustra, op. cit., p. 128.

12. Albert William Levi. Philosophy as Social Expression, Chicago, University of Chicago Press, 1974.

13. Peter Sloterdijk. Critique de la raison cynique, op. cit., p. 28.

14. Ibid., p. 27.

15. Ibid., p. 116. Traduction remaniée. 
16. Ibid., p. 15.

17. Idem.

18. Les résistances d'Adorno sur l'idée benjamienne du "corps humain comme mesure de la concrétude" sont mises au jour dans le texte d'Angela Cozea, dans ce même numéro, qui cite la correspondance Adorno-Benjamin pour témoigner de leur désaccord sur ce thème.

19. Peter Sloterdijk. Critique de la raison cynique, op. cit., p. 16.

20. Ibid., p. 19.

21. Ibid., p. 110.

22. Ibid., p. 27.

23. Peter Sloterdijk. La mobilisation infinie, trad. Hans Hildenbrand, Paris, Bourgois / Seuil, p. 98.

24. Peter Sloterdijk. Critique de la raison cynique, op. cit., p. 607.

25. Ibid., p. 31 .

26. Ibid., p. 43.

27. Ibid., p. 20-21.

28. Ibid., p. 149-150.

29. Ibid., p. 574.

30. Ibid., p. 17.

31. «À présent science, art et philosophie croissent en moi simultanément, au point que, de toute manière, j'engendrerai quelque jour un Centaure", in $F$. Nietżsche, Correspondance, G. Colli et M. Montinari (dir.), trad. J. Bréjoux et M. de Candillac, Paris, Gallimard, vol. 2, 1986, p. 93.

32. En 1986, Sloterdijk se consacre à une exégèse de Nietzsche en tant qu'auteur centauresque (Der Denker auf der Bühne. Nietzsches Materialismus, Frankfurt am Main, Suhrkamp, 1986. En français : Le penseur sur scène, trad. Hans Hildenbrand, Paris, Bourgois, 1990).

33. Peter Sloterdijk. Critique de la raison cynique, op. cit., p. 273.

34. Idem.

35. Titre original : Eurotaoismus. Zur Kritik der politischen Kinetik, Frankfurt am Main, Suhrkamp, 1989.

36. Peter Sloterdijk. La mobilisation infinie, op. cit., p. 24.

37. Ibid., p. 15. Je souligne.

38. Ibid., p. 126. Traduction remaniée.

39. Sjoerd van Tuinen. Peter Sloterdijk. Ein Profil, op. cit., p. 42. Ma traduction. Je souligne.

40. Peter Sloterdijk. La mobilisation infinie, op. cit., p. 181.

41. À noter que Heidegger est de loin l'auteur le plus cité dans Sphères I. Bulles, trad. Olivier Mannoni, Paris, Pauvert, 2002 et Sphères III. Écumes, trad. Olivier Mannoni, Paris, Maren Sell, 2005.

42. Theodor W. Adorno, 1931. "Die Aktualität der Philosophie», trad. anglaise, in The Adorno Reader, Oxford, Blackwell, 2000.

43. Jürgen Habermas. “Mit Heidegger gegen Heidegger denken». Frankfurter Allgemeine Zeitung, 25.7.1953. En français: «Penser avec Heidegger contre 
Heidegger?», in J. Habermas, Profils philosophiques et politiques, trad. Françoise Dastur et al., Paris, Gallimard, 1987.

44. Peter Sloterdijk enseigne, depuis 1992, à l'École des beaux-arts de Karlsruhe dont il est devenu le recteur en 2001. Cette position matérielle iconoclaste lui assure une liberté intellectuelle rare, certainement extra-universitaire.

45. Pierre Bourdieu. L'ontologie politique de Martin Heidegger, Paris, Minuit, 1988, p. 57.

46. Otto Kallscheuer. "Spiritus Lector. Die Zerstreuung des Zeitgeistes», in O. Kallscheuer et al., Peter Sloterdijks "Kritik der zynischen Vernunft", Frankfurt am Main, Suhrkamp, 1987, p.38. Ma traduction.

47. Ibid., p. 34-35.

48. Jürgen Habermas. "Zwischen Heine und Heidegger. Ein Renegat der Subjektphilosophie?», Die neue Unübersichtlichkeit, Frankfurt am Main, Suhrkamp, p. 121. Ma traduction.

49. Idem.

50. Voir les contributions de Daniel Jacques et Sébastien Mussi qui mettent au jour, dans ce numéro, le propos de Sloterdijk développé dans la conférence Règles pour le parc humain, mais à partir d'un horizon idéologique et exégétique distinct.

51. Le scandale du scandale a trait au fait que Habermas a enjoint Thomas Assheuer, directeur des pages culturelles du journal Die Zeit, d'attaquer le «projet Zarathoustra" de Sloterdijk (Thomas Assheuer. "Das Zarathustra-Projekt. Der Philosoph Peter Sloterdijk fordert eine gentetechnische Revision der Menscheit», Die Zeit, 2.9.99) qui «envisage froidement les potentialités diaboliques de la recherche génétique". Habermas est le réel instigateur de la polémique, car il a suggéré les invectices contre ce «jargon nazi» et cette "l'anthropologie des années 40 » dans une note secrète datée du mois d'août et rendue publique par la suite (Klaus-Gert Giesen. “Une question d'eugénisme en Allemagne», 2002, http://multitudes.samizdat.net ). II y est donc question non seulement de la réputation intellectuelle personnelle de Jürgen Habermas, mais aussi d'une dénonciation du sort réservé à un courant honni par la pensée allemande officielle : la pensée nietzschéenne et heideggerienne

52. Voir également Michel Kauffmann. «Le débat Sloterdijk-Habermas de l'automne 1999 : une méta-polémique?», in Valérie Robert (éd.), Intellectuels et polémiques dans l'espace germanophone, Paris, Publications de l'Institut d'Allemand, 2003, p. 161-174.

53. Otto Kallscheuer. «Spiritus Lector. Die Zerstreuung des Zeitgeistes», op. cit., p. 27. Ma traduction.

54. Sjoerd van Tuinen. Peter Sloterdijk. Ein Profil, op. cit., p. 126. Ma traduction.

55. Peter Sloterdijk. «Dankesrede. Sigmund-Freud Preis» : www.petersloterdijk.net

56. Dans une conférence, « Sloterdijk as an Alternative to the Nature/Culture Division " (Bruxelles, 23 février 2007), Bruno Latour, ami et grand partisan de Sloterdijk, stipule à ce titre que l'œuvre doit être prise au sérieux par les sciences sociales, car elle respatialise et rematérialise le "social» contre l'abstraction réifiante. La "société» de Hitler, par exemple, devient ici la sonosphère, c'est-à-dire le rassemblement de Nuremberg dont la limite est marquée par l'écho de la voix amplifiée du Führer.

57. Henk Oosterling. “Interest and Excess of Modern Man's Radical Mediocrity», Cultural Politics, 3/2007. À paraître.

58. Cette posture, qui pose des limites certaines, fera aussi dire à Latour que Sloterdijk 
n'hésite jamais dans son discours, il ne pèse aucune possibilité, aucune avenue, il ne compare aucune ligne argumentative, il ne formule aucune hypothèse. II est narrateur-Dieu.

59. Peter Sloterdijk. Le palais de cristal. À l'intérieur du capitalisme planétaire, trad. Olivier Mannoni, Paris, Maren Sel, 2006, p. 375.

60. Henk Oosterling. «Interest and Excess of Modern Man's Radical Mediocrity». Ma traduction.

61. Sjoerd van Tuinen. Peter Sloterdijk. Ein Profil, op. cit., p. 157. Ma traduction.

«Je tiens à remercier Sjoerd van Tuinen, Koenraad Hemelsoet, Henk Oosterling, Frank Hartmann, Robert Pfaller, Marc Jongen et Bruno Latour qui, lors du colloque Measuring the Monstrous: Peter Sloterdijk's Jovial Modernity (Bruxelles, 23 février 2007), ont généreusement contribué à nourrir ma lecture de l'œuvre de Peter Sloterdijk. Que la réception continue...» 